\title{
أثز السياسات السعرية على محصول البطاطس في مصر
}

\author{
مها محمد عليوة \\ قسم الاقتصاد و إدارة الاعمال الزر اعية- كلية الزر اعة- جامعة الاسكندرية
}

\section{الملخص}

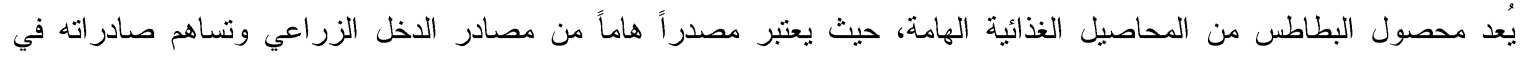
الحصول على النقد الأجنبي، ولذلك تتحصر مشكلة البحث في أثز التنخل الحكومى المباثر أو غير المباثر فى السياسات الزراعية التبعيرية

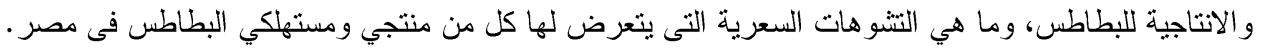

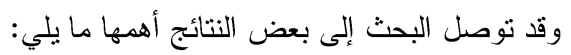

( () المؤشر ات الإنتاجية المنتلة في كل من المساحة المزروعة والإنتاجية الفدانية والإنتاج الكلي و الاستهلالك لمحصول البطاطس خلال الفترة

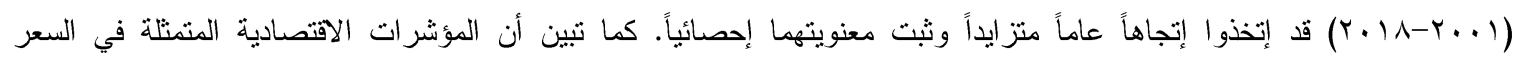

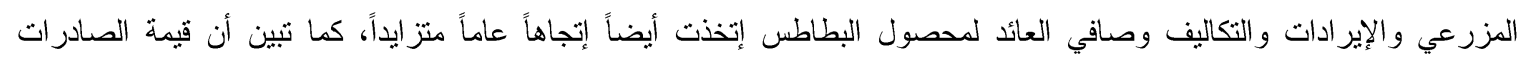

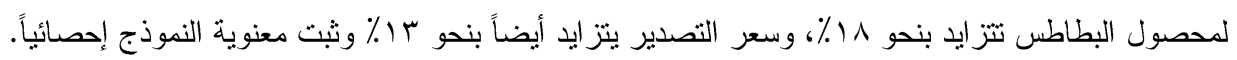

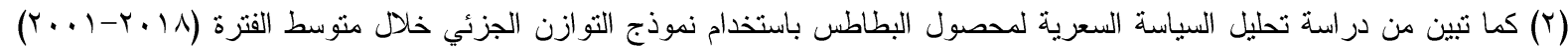

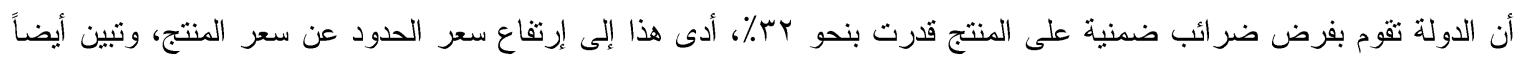
فرض ضر ائب على المستهلك، ولذلك تكون السياسة المتبعة خلال فترة الدراسة في غير صالح المستهلك أدى ذلك إلى إرتفاع أسعار التجزئة عن أسعار الحدود.

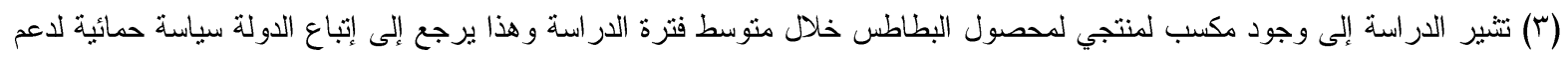

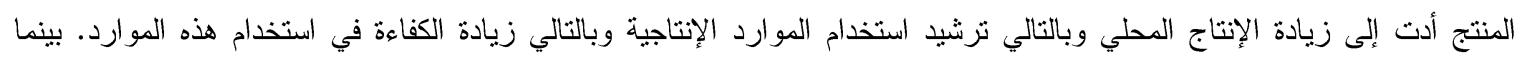

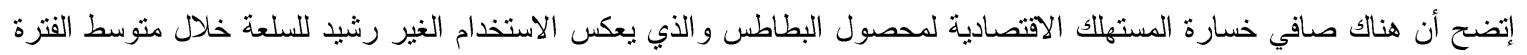

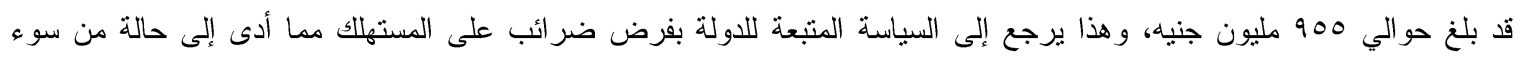

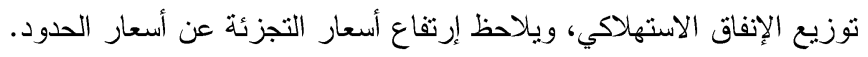

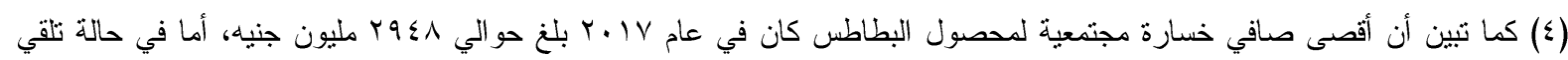

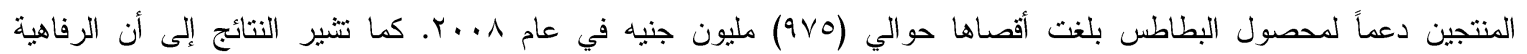

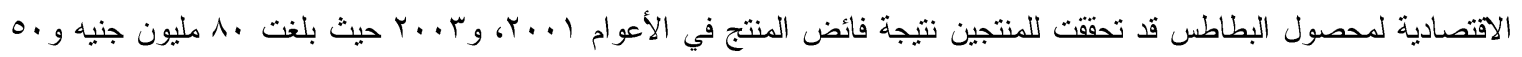

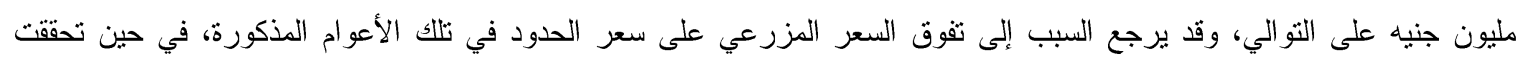

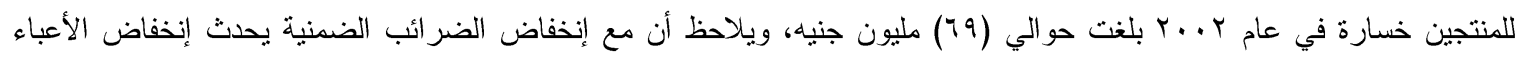

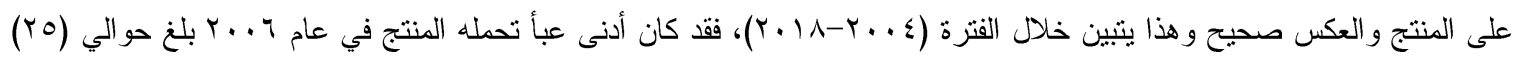

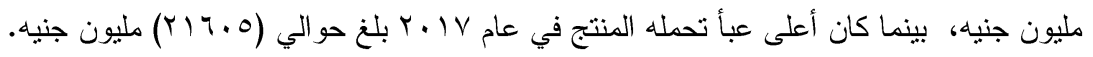

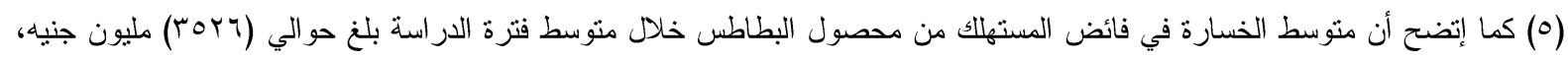

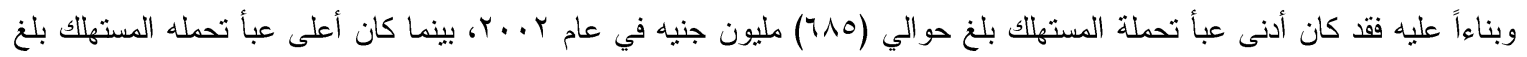

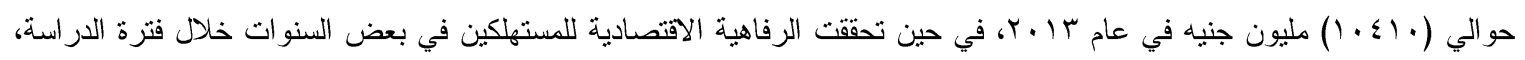

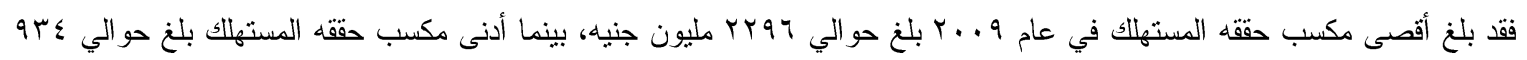

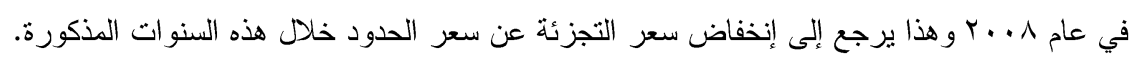

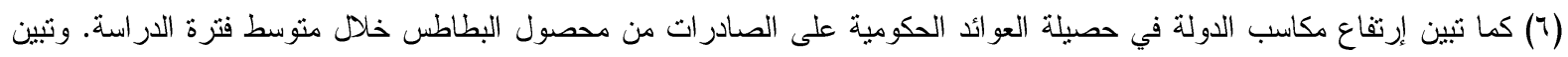

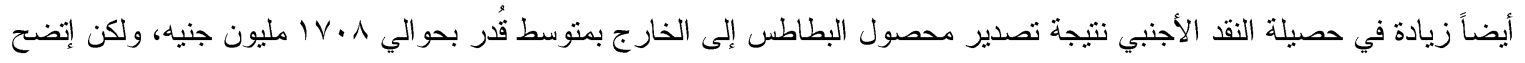

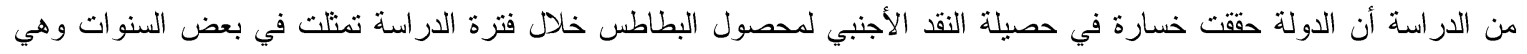

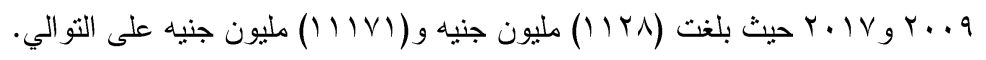




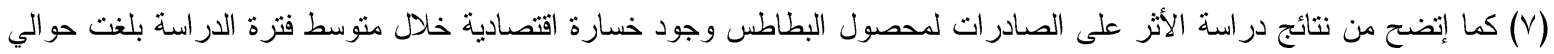

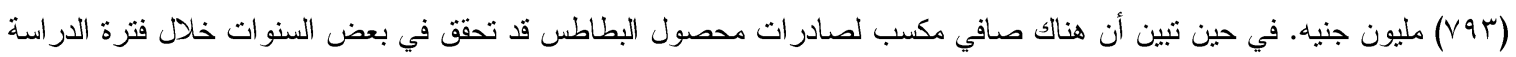

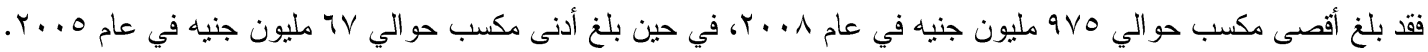

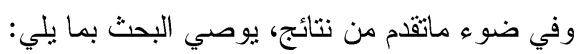
(1) ضرورة دراسة وتعديل السياسات المنعلقة بدعم المستهلك، والتي أدت إلى استخدام غير رشيد لمحصول البطاطس وإلى حالة من سوء توزيع الإنفاق الاستهلاكي.

(r) الإستمرار في مراجعة وتعديل برنامج الإصلاح الاقتصادي خاصة فيما يتعلق بمعالجة الآثار السلبية للبرنامج والخاصة بتخفيض الضر ائب المفروضة على المنتجين الزراعيين، والتي تؤدي زيادتها إلى زيادة صافي الخسارة الاقتصادية في إنتاج محصول البطاطس.

\section{الكلمات المفتاحية: السياسة السعرية، الميزان التجاري، نموذج الثوازن الجزئي.}

\section{المشكلة البحثية}

نظر اً لمكانة محصول البطاطس في الإنتاج

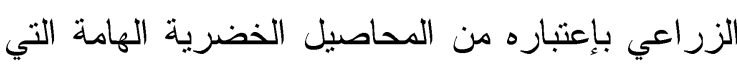
يتم زراعتها في ثلاث عروات، حيث نمنئ تمنل الأهمية

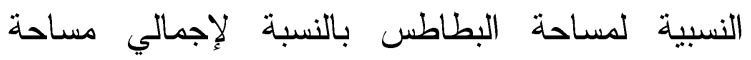

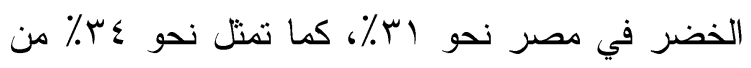

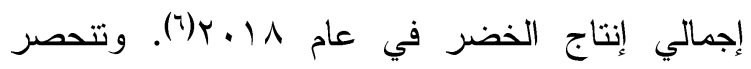
مشكلة البحث في أثز التدخل الحكومي المباشر أو غير فير المباشر فى السياسات الزراعية التسعيرية والانتاجية

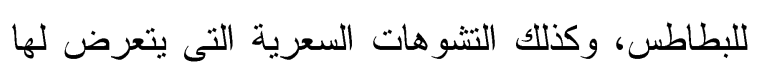
كل من منتجي ومستهلكي البطاطس فى مصر، وما لها

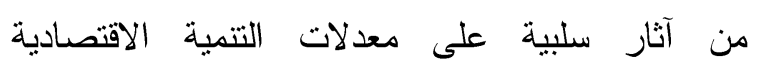
و الاجتماعية في القطاع الزر اعي، التي تؤدي إلى عدم كفاءة تخصيص الموارد الاقتصادية لقطاع الزراعة.

\section{أهداف البحث}

يتمثل الهدف الرئيسي للبحث فــي دراســة أثــر

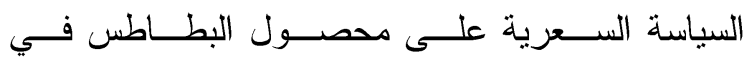

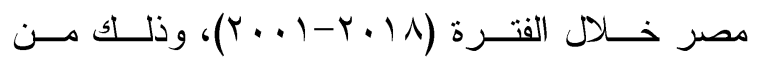

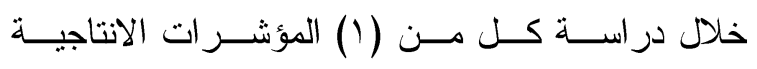

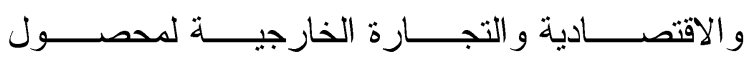
البطاطس، (Y) اســتخدام نــــوذج التــوازن الجزئسـي لقياس أثر تطبيق سياسة التدخل الحكومي.

\section{المقدمة}

يُعد محصول البطاطس أحد محاصيل الخضر الغذائية و التصنيعية الإستر اتيجية الهامة في مصر ، حيث لهُبن تقوم على هذا المحصول بعض الصناعات الغذائية بإعنباره من أهم المحاصيل النشوية حيث بلغ إنتاج

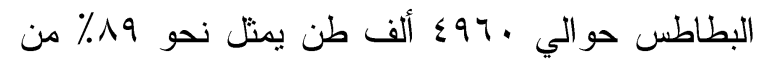

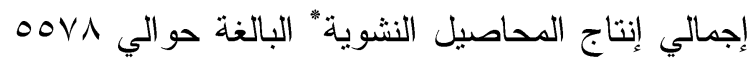
ألف طن في عام 1 1.ب(?)، ويعتبر هذا المحصول

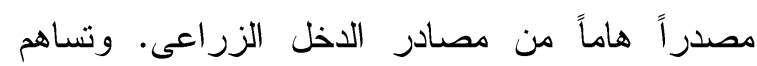
صادرات البطاطس في الحصول على النقد الأجنبي حيث بلغت كمية وقيمة صادرات البطاطس حوالي لبط S NV

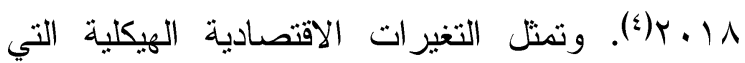
شهدها الإقتصاد المصري عقب تطبيق سياسات

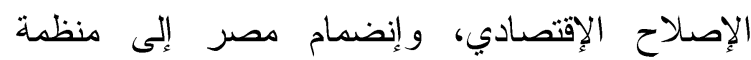

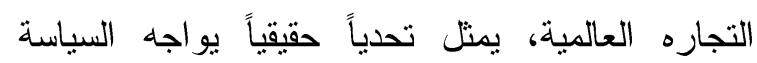

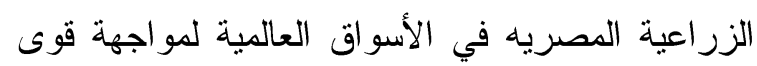

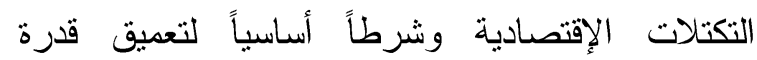
الزراعة المصرية التنافسية للحد من الرسوم الجمركية

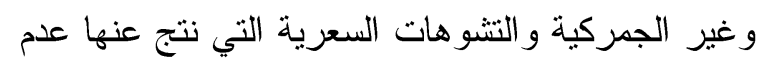
كفاءة تخصيص الموارد. " عبارة عن (البطاطس، القلقاس، البطاطا). 
Change in Consumer Surplus

$$
\text { (ب) التغير في فائض المستهلك (CS): }
$$

$\mathrm{C}_{\mathrm{S}}=\mathrm{C}_{\mathrm{d}} \times\left(\mathrm{P}_{\mathrm{b}}-\mathrm{P}_{\mathrm{d}}\right)-\mathrm{NEL}_{\mathrm{C}}$

$$
\text { (ع ) حصيلة عوائد الدولة: }
$$

(أ) التغير في حصيلة النقد الأجنبي(FE):

Change in Foreign Exchange

$\mathrm{FE}=-\mathrm{P}_{\mathrm{b}} \times\left(\mathrm{Q}_{\mathrm{W}}-\mathrm{Q}_{\mathrm{d}}+\mathrm{C}_{\mathrm{d}}-\mathrm{C}_{\mathrm{W}}\right)$

$$
\text { (ب)(التغير في الإير اد الحكومي(GR): }
$$

Change in Government Revenue

$\mathrm{GR}=\mathrm{Q}_{\mathrm{d}} \times\left(\mathrm{P}_{\mathrm{b}}-\mathrm{P}_{\mathrm{d}}\right)-\mathrm{C}_{\mathrm{d}} \times\left(\mathrm{P}_{\mathrm{b}}-\mathrm{P}_{\mathrm{d}}\right)$

$=-\mathrm{NEL}-\mathrm{P}_{\mathrm{S}}-\mathrm{C}_{\mathrm{S}}$

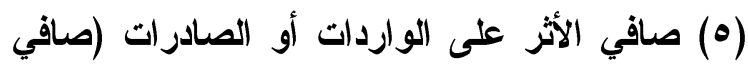

$$
\begin{aligned}
& \text { (المكاسب أو الخسائر الأقتصادية) (NET): }
\end{aligned}
$$

Net Effect in Exports or Imports

$\mathrm{NET}=\mathrm{GR}+\mathrm{P}_{\mathrm{S}}+\mathrm{C}_{\mathrm{S}}$

$$
\begin{aligned}
& \text { حيث أن: } \\
& \text { (سعر المنتج أو سعر المستهلك) Pd }
\end{aligned}
$$

Domestic Farm Gate Price

Border price سعر الحدود $\quad$ = Pb

Production at Border Price

$$
\text { كمية الإنتاج عند سعر الحدود }=\text { Qw }
$$

$$
\text { = Sمية الإنتاج عند السعر المحلي }=
$$

Production at Domestic Farm Gate Price

$$
\text { كمية الاستهلاك عند سعر الحدود }=C_{W}
$$

Consumption at Border Price

$$
\text { Cd }
$$

Consumption at Domestic Farm Gat Price

وقد إعتمد البحث على البيانات الثانوية الصادرة من

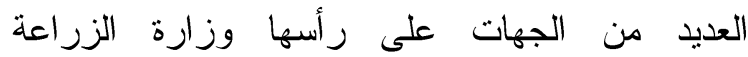

و إستصدلاح الأراضي، والجهاز المركزي للتعبئة العامة

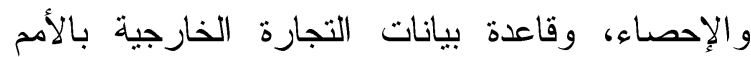

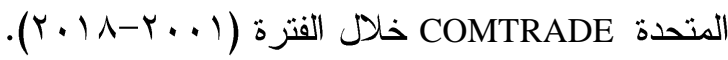

\section{النتائج البحثية ومناقشتها}

أولاً: المؤشرات الإتتاجية والاقتصادية والتجارة الخارجية لمحصول البطاطس في مصر:

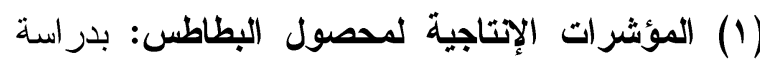

المؤشرات الإنتاجية لمحصول البطاطس في مصر الإنه

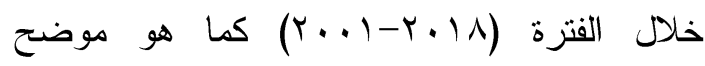

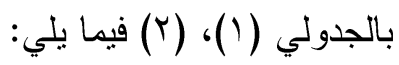

\section{الأسلوب البحثي ومصادر البيانات}

اعتمد البحث في تحقيق أهدافة علــى اســـخدام

التحليل الاقتصادي الوصفي و الكمي، وذلك من خـلال

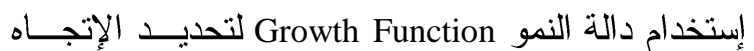
العام ومعدل التغير للمتغيرات موضع الدراسة، وكذلك

استخدام نموذج التوازن الجزئسي

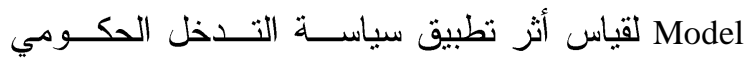

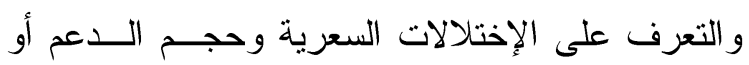

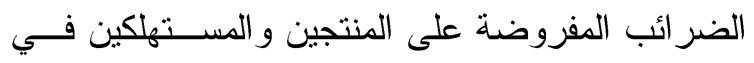
سوق سلعة معينة، وأثر ذلك على الرفاهية الاقتصـادية

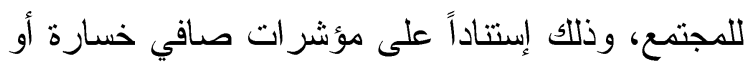
مكسب كل من المنتج و المستهلك الاقتصادية، و التغيـر

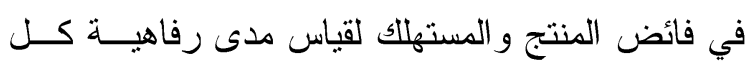
منهما، و أثر تلك السياسة على الإيراد الحكــومي مــن فئن

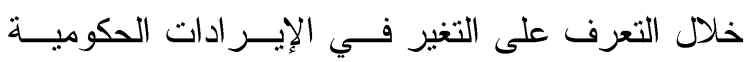

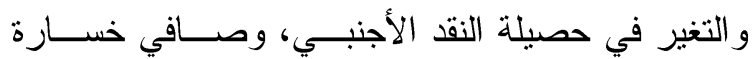

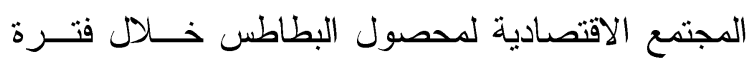

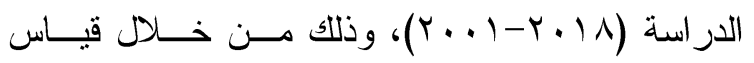

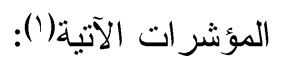

(1) مؤشرات الحماية السعرية:

Nominal Protection Coefficient (أ) معامل الحماية الاسمي (NPC): $\mathrm{NPC}=\mathrm{Pd} / \mathrm{Pb}$

(Y) مؤشرات الكفاءة الاقتصادية:

(أ) صافي الخسارة على مستوى المنتج (NELP):

Net Economic Loss in Production $\mathrm{NEL}_{\mathrm{P}}=0.5\left(\mathrm{Q}_{\mathrm{w}}-\mathrm{Q}_{\mathrm{d}}\right) \times\left(\mathrm{P}_{\mathrm{b}}-\mathrm{P}_{\mathrm{d}}\right)$

(ب) صافي الخسارة على مستوى المستهلك (NELC)

Net Economic Loss in Consumption

$\mathrm{NEL}_{\mathrm{C}}=0.5\left(\mathrm{C}_{\mathrm{w}}-\mathrm{C}_{\mathrm{d}}\right) \times\left(\mathrm{P}_{\mathrm{b}}-\mathrm{P}_{\mathrm{d}}\right)$

$$
\text { (ج) صافي الخسارة على مستوى المجتمع (NEL): }
$$

Total Net Economic Loss

$\mathrm{NEL}=\mathrm{NEL}_{\mathrm{P}}+\mathrm{NEL}_{\mathrm{C}}$

Change in Producer surplus

$$
\text { (أ) التغير في فائض المنتج (PS): }
$$

$\mathrm{P}_{\mathrm{S}}=\mathrm{Q}_{\mathrm{d}} \times\left(\mathrm{P}_{\mathrm{d}}-\mathrm{P}_{\mathrm{p}}\right)-\mathrm{NEL}_{\mathrm{P}}$ 


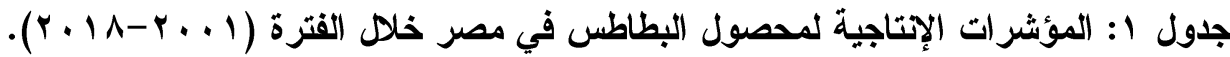

\begin{tabular}{|c|c|c|c|c|}
\hline 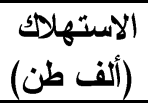 & الإلتتاج الكلي & الإتتاجية الفدانية & المساحة المزروعة فانة) & السنة \\
\hline $1 \pi \leqslant r$ & $19 . r$ & $1 \cdot, \cdot r$ & 19. & $r \ldots 1$ \\
\hline 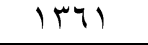 & 1910 & $1 \cdot, 1$. & $19 \mathrm{~V}$ & $r \ldots r$ \\
\hline $1 T \leqslant 1$ & $r . r q$ & $1 \cdot, r \varepsilon$ & $19 V$ & $r \ldots r$ \\
\hline 1049 & ro $\leqslant V$ & $1 \cdot, Y V$ & $r \leqslant \Lambda$ & $r \ldots \varepsilon$ \\
\hline YYYO & MITV & $1 ., 0 r$ & $r .1$ & r...o \\
\hline $1 \leqslant 0$. & TrIT & $1 \cdot, 0$. & Kr. & $r \ldots r$ \\
\hline$I V \leqslant r$ & rVY. & $1 \cdot, v \varepsilon$ & rOV & $r \ldots v$ \\
\hline FYTY & TOTV & $1 \cdot, 19$ & TYV & $r \ldots \Lambda$ \\
\hline$r r . q$ & 1409 & $11,1$. & .r. & $r \ldots q$ \\
\hline$r r . q$ & 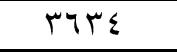 & $1 \cdot$, , T & סטז & $r .1$. \\
\hline rol. & $\varepsilon r \mu \wedge$ & $11,1$. & r91 & 5.11 \\
\hline rAYT & $\varepsilon v 01$ & $11, Y \wedge$ & $\sum Y r$ & $r .1 r$ \\
\hline ro.r & $\sum Y Y O$ & 11,11 & rNI & $r .1 T$ \\
\hline Yols & $\{711$ & $11, Y_{4}$ & $\leqslant 1$. & $r .1 \varepsilon$ \\
\hline 1910 & $\sum 900$ & rr, & $\varepsilon r V$ & $r .10$ \\
\hline$r \leqslant V 0$ & $\varepsilon \| 1 \pi$ & $1 \cdot, 9 Y$ & TVV & 5.17 \\
\hline r700 & $\varepsilon \wedge \leqslant 1$ & $11,4 \mathrm{~V}$ & $\leqslant 10$ & $Y . I V$ \\
\hline$r V \leq T$ & $\sum 97$. & $1 Y, 10$ & $\varepsilon \cdot 1$ & $r .11$ \\
\hline 5179 & rova & $1 \cdot, 9$. & Tro & المتوسط \\
\hline ro & 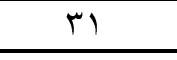 & 0 & $r V$ & معامل الإختلاف \\
\hline
\end{tabular}

المصدر : جمعت وحسبت من:

- وزارة الزر اعة وإستصلاح الأراضى، قطاع الثئون الاقتصادية، نثرة الإحصاءات الزر اعية، أعداد متفرقة.

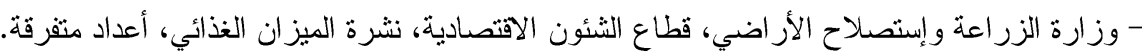

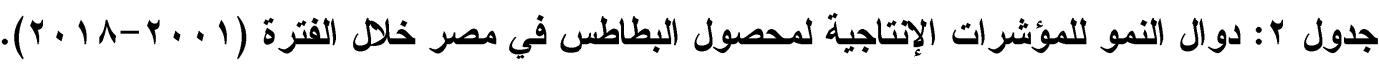

\begin{tabular}{|c|c|c|c|c|}
\hline معدل النمو(\%) & $\mathbf{F}$ & $\mathbf{R}^{2}$ & المعادله & المتغير \\
\hline 5 & $(81.32)^{* *}$ & 0.84 & $\begin{aligned} \operatorname{Ln} \hat{Y}_{\mathrm{i}}=5.269+0.050 \mathrm{~T}_{\mathrm{i}} \\
(87.71)^{* * *} \quad(9.02)^{* *}\end{aligned}$ & 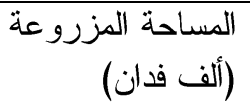 \\
\hline 0.9 & $(105.46)^{* *}$ & 0.87 & $\begin{aligned} \operatorname{Ln} \hat{Y}_{\mathrm{i}}=2.304+0.009 \mathrm{~T}_{\mathrm{i}} \\
(248.02)^{* *} \quad(10.27)^{* *}\end{aligned}$ & $\begin{array}{r}\text { الإنتاجية الفدانية } \\
\text { (طدان) }\end{array}$ \\
\hline 5.9 & $(102.66)^{* * *}$ & 0.87 & $\begin{array}{l}\operatorname{Ln} \hat{Y}_{i}=7.573+0.059 T_{i} \\
\quad(120.36)^{* * *} \quad(10.13)^{* *}\end{array}$ & $\begin{array}{l}\text { الإنتاج الكلي طن) } \\
\text { (ألف }\end{array}$ \\
\hline 4.6 & $(56.76)^{* *}$ & 0.78 & $\begin{aligned} \operatorname{Ln} \hat{Y}_{i} & =7.209+0.046 T_{i} \\
(108.65)^{* *} & (7.53)^{* *}\end{aligned}$ & 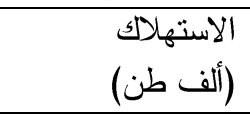 \\
\hline
\end{tabular}

المصدر : حُسبت من نتائج التحليل الإحصائي للبيانات الو اردة بالجدول(1). 
أقصى بلغ حوالي 910 ألف طن عام 10 ب.

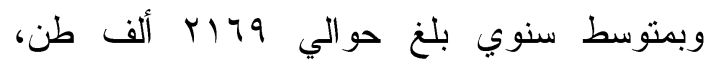
وبمعامل إختلاف قدر بنحو هب ٪، وبتقدير دالة النمو

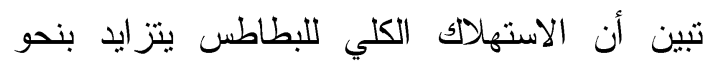

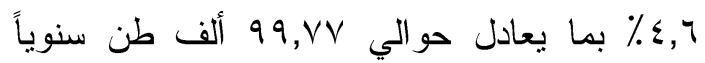
خلال فترة الدراسة، وهو تز ايد معنوي إحصائياً عند مستوى معنوية إ., •. (ب) المؤشرات الإقتصادية لمحصول البطاطس في مصر: بدراسة المؤشرات الاقتصادية للمحصول

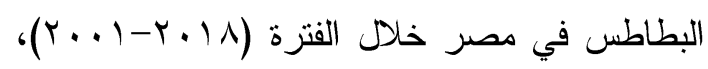
كما هو موضتح بجدولي (r)، (ع) فيما يلي:

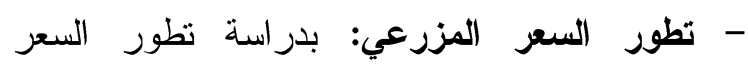
المزرعي لمحصول البطاطس تنين أنه تزاوح بين لطورين

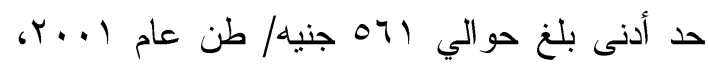

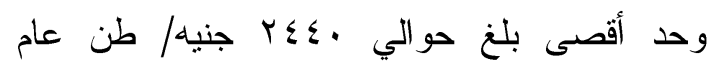

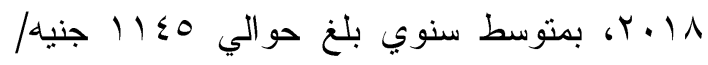
طن، وبمعامل إختلاف قدر بنحو ؟ § ٪، وبتقدير دالة النمو تبين أن السعر المزرعي للبطاطس يتزايد

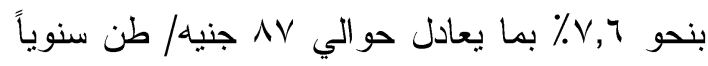
خلال فترة الدر اسة، وهو تز ايد معنوي إحصائياً عند مستوى معنوية l +, •. - تطور الإيرادات الكلية: بدراسة نطور الإيرادات

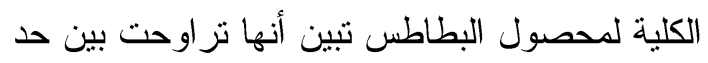

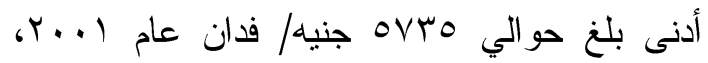

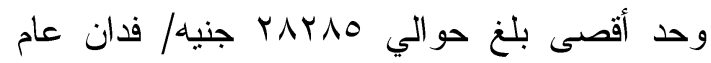

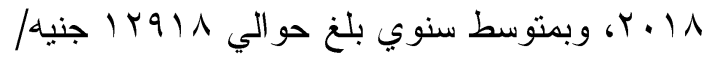
فدان، وبمعامل إختلاف قدر بنحو 7٪ء؛ وبتقدير دالة النمو تبين أن الإيرادات الكلية للبطاطس تنز ايد إنداب

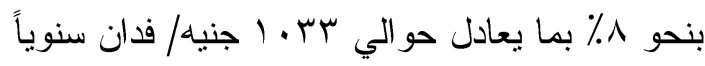
خلال فترة الدر اسة، وهو تز ايد معنوي إحصائياً عند

$$
\text { مستوى معنوية ا +, •. }
$$

- تطور المساحة المزروعة: بدراسة تطور المساحة

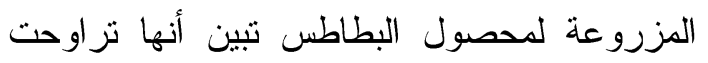

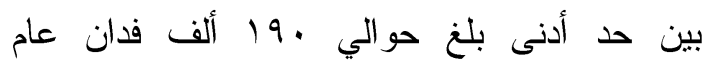

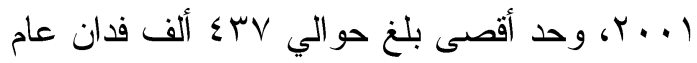

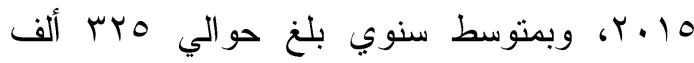

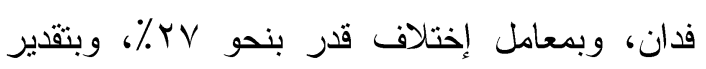
دالة النمو تبين أن المساحة المزروعة للبطاطس

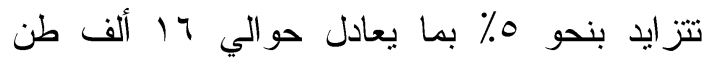

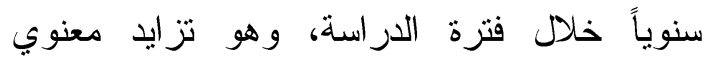

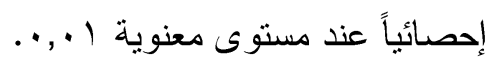
- تطور الإنتاجية الفداتية: بدراسة تطور الإنتاجية

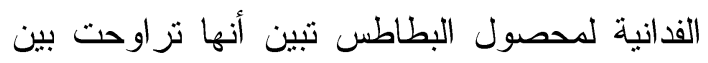

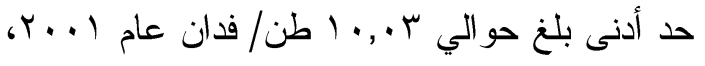

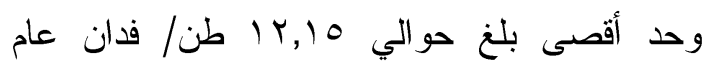

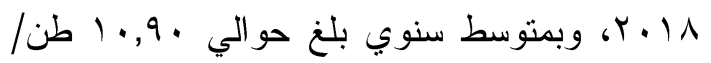

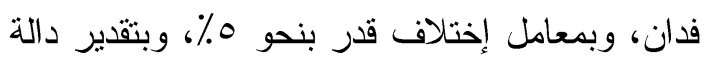

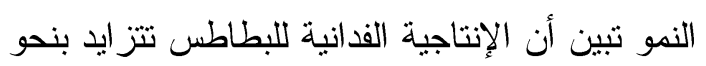

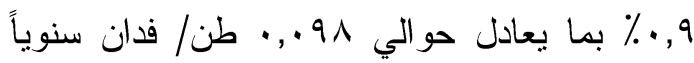

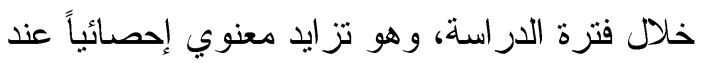

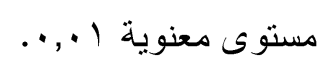
- تطور الإنتاج الكلي: بدراسة تطور الإنتاج الكلي

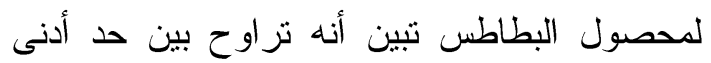

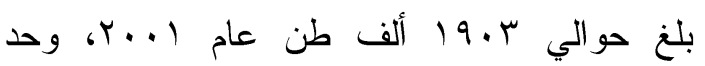

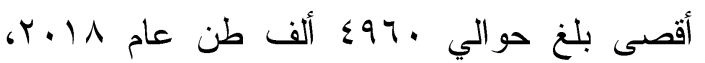

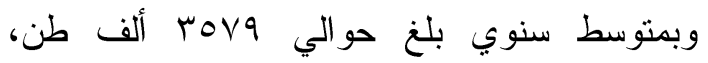
وبمعامل إختلاف قدر بنحو آسا، وبتقدير دالة النمو تبين أن الإنتاج الكلي للبطاطس يتزايد بنحو

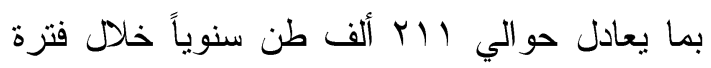

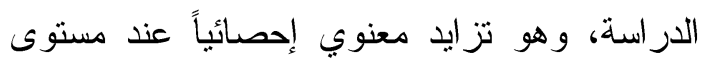
معنوية ال, •..

- تطور الاستهلاك الكلي: بدراسة تطور الاستهلاك الكلي لمحصول البطاطس تبين أنه نراوح بين حد لاسنهون

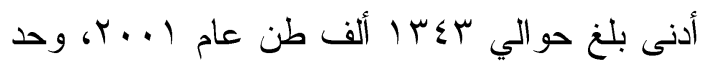


وبمعامل إختلاف قدر بنحو 101 و، وبتقدير دالة النمو

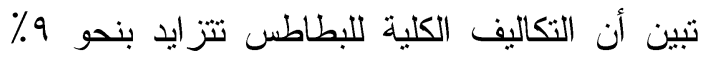
بما يعادل حو الي V99 جنيد/ فدان سنوياً خلال فترة الدراسة، وهو تزايد معنوي إحصائياً عند مستوى لماديان

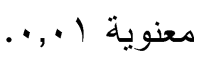

- تطور التكاليف الكلية: بدراسة تطور التكاليف الكلية

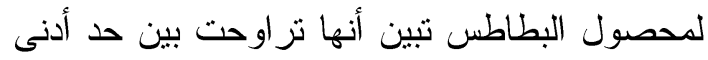

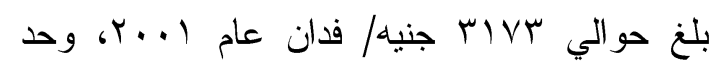

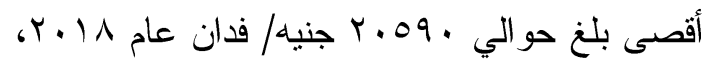
وبمتوسط سنوي بلغ حوالي ANVA جنيه/ فدان،

جدول r: المؤشرات الاقتصادية لمحصول البطاطس في مصر خلال القترة (1 . . ץ-1 I • ؟).

\begin{tabular}{|c|c|c|c|c|}
\hline (جنية/ فافان) & التكاليف (الكلية & الإيزيله/ فدان) الكلية & السعر المزرعي طن) & السنة \\
\hline roTr & rIVr & OVTO & 071 & $r \ldots l$ \\
\hline TYTT & ro0. & 0117 & OVY & $r \ldots r$ \\
\hline r701 & $\{109$ & $7 N I V$ & $7 \leqslant 7$ & $r \ldots r$ \\
\hline $171 \pi$ & OYO0 & 7179 & Tr. & r... \\
\hline $1 \leqslant \varepsilon r$ & $0 \leqslant 9 Y$ & $794 \varepsilon$ & $T Y \varepsilon$ & r...o \\
\hline YTYS & 7190 & 1119 & $\vee \wedge \varepsilon$ & $r \ldots r$ \\
\hline 0.1. & $V 1.0$ & $1 Y 110$ & $1 . \leqslant r$ & $r \ldots V$ \\
\hline$\varepsilon ॉ$ हाए. & 101. & IrTrq & $1 . A V$ & $r \ldots$ \\
\hline$\sum 990$ & $\Lambda V .0$ & 111799 & 119. & $r \ldots q$ \\
\hline 0.19 & NOYT & $1+41 \pi$ & TYYT & r.1. \\
\hline $7 \wedge \leqslant 0$ & $9 Y .1$ & $17 . \leqslant 7$ & ITAT & $r .11$ \\
\hline rove & $1 \ldots r \varepsilon$ & 11411 & $11 \wedge r$ & $r .1 Y$ \\
\hline$r \leqslant 09$ & 1.197 & 11400 & IYYT & $r .1 r$ \\
\hline$\sum r 00$ & $9 \leqslant 01$ & $1 \mu \Lambda .7$ & $1 r r q$ & $r .1 \varepsilon$ \\
\hline हाग० & 9074 & 117991 & $T Y \varepsilon$ & $r .10$ \\
\hline$\{001$ & $|r Y q|$ & $17 \lambda \leqslant \Lambda$ & $100 Y$ & $r .17$ \\
\hline $07 r \varepsilon$ & IVAIY & $r \Psi \leq \leqslant 7$ & 191. & $r .1 V$ \\
\hline 17990 & $r .09$. & rAYAO & $r \leqslant \varepsilon$. & $r .11$ \\
\hline$\varepsilon \cdot \varepsilon$. & $\Lambda \wedge \vee \wedge$ & $1 Y 9 \mid 1$ & $11 \leq 0$ & المتوسط \\
\hline$\varepsilon r$ & 01 & $\sum 7$ & $\leqslant \varepsilon$ & معامل الإختلاف \\
\hline
\end{tabular}

- وزارة الزراعة وإستصلاح الأراضى، قطاع الثئون الاقتصادية، نشرة النكاليف وصافي العائد، أعداد متفرقة.

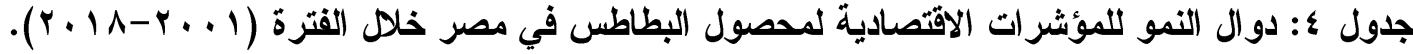

\begin{tabular}{|c|c|c|c|c|}
\hline معدل النمو(\%) & $\mathbf{F}$ & $\mathbf{R}^{2}$ & المعادله & المتغير \\
\hline 7.6 & $(134.63)^{* *}$ & 0.89 & $\begin{array}{r}\operatorname{Ln} \hat{Y}_{i}=6.240+0.076 \mathrm{~T}_{\mathrm{i}} \\
(88.58)^{* * *} \quad(11.60)^{* * *}\end{array}$ & السعر المزرعي(جنيه/طن) \\
\hline 8 & $(113.14)^{* *}$ & 0.88 & $\begin{aligned} \operatorname{Ln} \hat{Y}_{i}=8.605+0.080 T_{i} \\
\quad(105.16)^{* * *} \quad(10.64)^{* * *}\end{aligned}$ & الإير ادات(جنيه/ فدان) \\
\hline 9 & $(174.33)^{* *}$ & 0.92 & $\begin{aligned} \operatorname{Ln} \hat{Y}_{i}=8.122+0.090 T_{i} \\
\quad(110.38)^{* * *}(13.20)^{* *}\end{aligned}$ & التكاليف(جنيه/ فدان) \\
\hline \multirow[t]{3}{*}{6.3} & $(18.11)^{* *}$ & 0.53 & $\begin{aligned} \operatorname{Ln} \hat{Y}_{i}=7.608+0.063 T_{i} \\
\quad(47.22)^{* * *} \quad(4.26)^{* *}\end{aligned}$ & صافي العائد(جنيه/ فدان) \\
\hline & & & 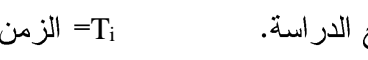 & Y = القيمة الثقديرية للمتغير التابع \\
\hline & &.$\cdot 1$ & 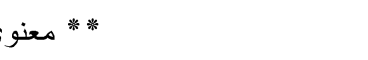 & $. \mid \wedge \ldots \ldots \ldots, r(1)=i$ \\
\hline
\end{tabular}


- تطور صافي العائد: بدراسة تطور صافي العائد (ج) مؤشرات التجارة الخارجية لمحصول البطاطس:

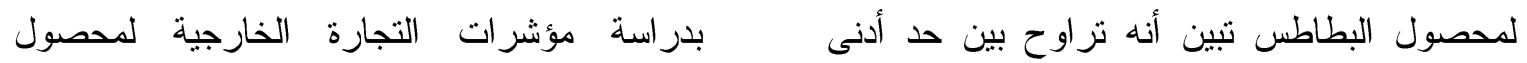

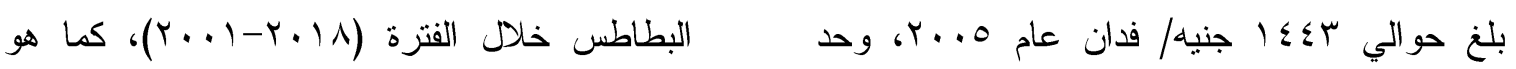

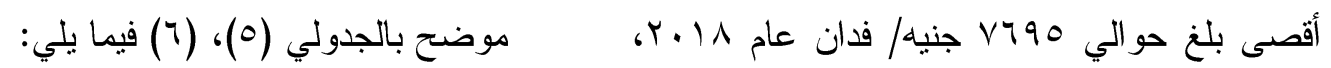

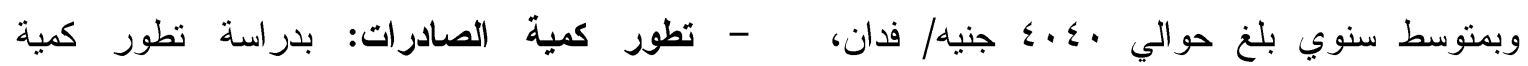

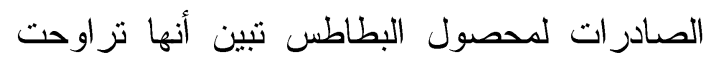

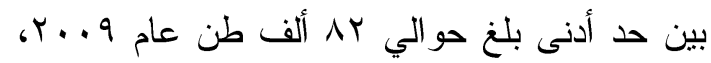

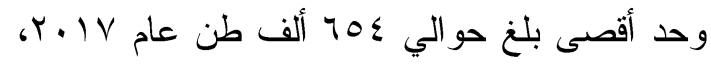

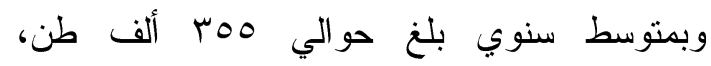

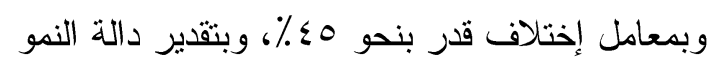

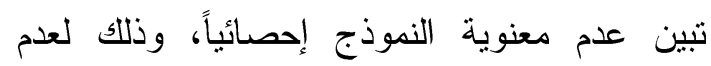

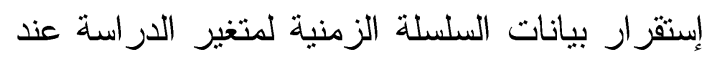

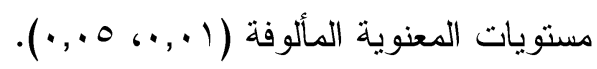

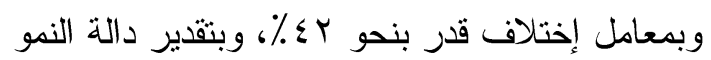

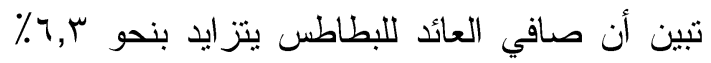

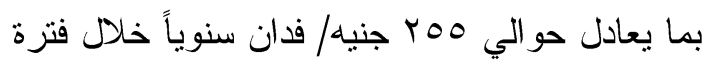

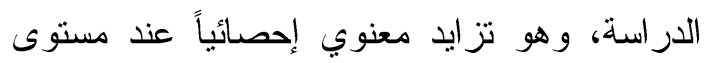
معنوية ا +, •.

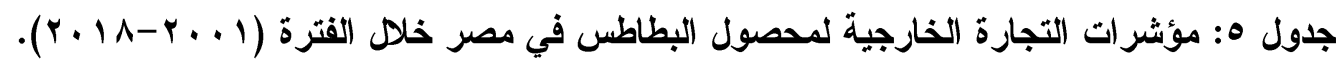

\begin{tabular}{|c|c|c|c|}
\hline سعر التصدير & قيمة الصادرات & كمئة الصنادرات & السنة \\
\hline$O \wedge \varepsilon$ & 1.1 & 110 & r... \\
\hline$V 99$ & $1 \wedge r$ & rYq & $r \ldots r$ \\
\hline$V \leqslant T$ & FYI & ra9 & $r \ldots r$ \\
\hline 1.11 & §) & rی. & r... \\
\hline $11 \pi$. & $\varepsilon \varepsilon 1$ & rq. & r...o \\
\hline $1.1 Y$ & $r v$. & ד4 & $r \ldots T$ \\
\hline 10rs & $0 \wedge V$ & rAr & $r \ldots v$ \\
\hline FYT9 & YIY & $9 \leqslant$ & $r \ldots h$ \\
\hline$r v_{\ldots}$ & $r \cdot r$ & NY & $r \ldots q$ \\
\hline TOYK & $7 \wedge 1$ & $T V T$ & r.l. \\
\hline $19 \Lambda Y$ & $V \cdot V$ & rov & r.11 \\
\hline r97A & $V Y q$ & $r \leqslant 7$ & $Y . / Y$ \\
\hline rrrq & 1181 & ror & $r .1 r$ \\
\hline$r \varepsilon \cdot \lambda$ & 1910 & 070 & T.I \\
\hline rqY. & 1794 & $0 \wedge$. & r.10 \\
\hline ro10 & $17 V V$ & $\sum 71$ & T.17 \\
\hline$V) \leqslant V$ & $\sum T V T$ & $70 \leqslant$ & T.IV \\
\hline $7 \leqslant \wedge 4$ & T171 & $\varepsilon \wedge V$ & $r .11$ \\
\hline TITK & $1 . V$. & roo & المتوسط \\
\hline V) & 114 & $\leqslant 0$ & معامل الإختلاف \\
\hline
\end{tabular}

www. COMTRADE.un.org المصدر : جمعت وحسبت من: قاعدة بيانات التجارة الخارجية بالأمم المتحدة 


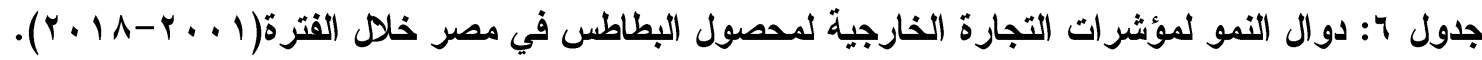

\begin{tabular}{|c|c|c|c|c|}
\hline معدل النمو (\%) & $\mathbf{F}$ & $\mathbf{R}^{2}$ & المعادله & المتغير \\
\hline 18 & $(99.92)^{* *}$ & 0.88 & $\begin{array}{r}\operatorname{Ln} \hat{Y}_{i}=4.757+0.180 T_{i} \\
(24.37)^{* *} \quad(10.00)^{* *}\end{array}$ & قيمة الصادر ات (مليون جنيه) \\
\hline 13 & $(129.43)^{* *}$ & 0.89 & $\begin{array}{r}\operatorname{Ln} \hat{Y}_{i}=6.398+0.130 \mathrm{~T}_{\mathrm{i}} \\
(51.78)^{* * *} \quad(11.38)^{* *}\end{array}$ & سعر التصدير (جنيه/طن) \\
\hline
\end{tabular}

المصدر : حسبت من نتائج التحليل الاحصائي للبيانات الو اردة بالجدول (0). - تطور سعر التصدير : بدراسة تطور سعر لإليل

المزرعي للمنتج إلى سعر الحدود للسلعة، وهو بذلك الكي

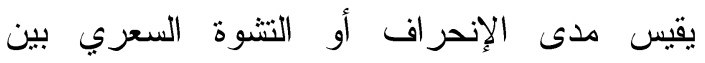
السعرين، فإذا كان معامل الحماية للمنتج أكبر من العندي الواحد الصحيح فهو يوضح وجود دعم داخلي معاني

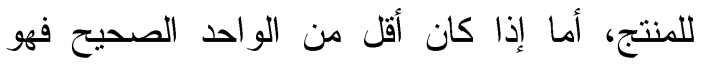
يوضح وجود ضرائب ضمنية على المنتج داخلياً، بينما إذا كان مساوياً للواحد الصحيح فهو يوضح فئح إتباع سياسة حيادية أي عدم فرض ضريبة أبان أو دعم

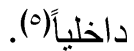

ويتضح من بيانات الجدول (^)، أن متوسط معامل الحماية الأسمي للمنتج لمحصول البطاطس في مصر

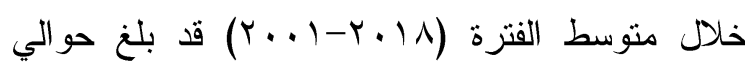
11،، إي أن منتجي محصول البطاطس قد حصلوا

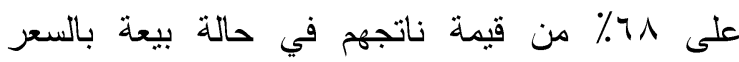
العالمي خلال فترة الدراسة، وهذا يعني أن الدولة نقوم بفرض ضرائب ضمنية على المنتج قدرت بنحو بـr\% من قيمة ناتجهم في حالة بيعة بالسعر العالمي، حيث

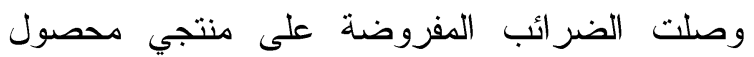

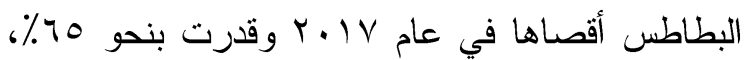

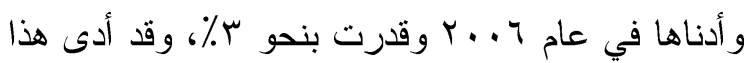
إلى إرتفاع سعر الحدود عن سعر المنتج. أما معامل الحماية الأسمي للمستهلك فيعبر عندر عن نسبة سعر التجزئة للمستهلك إلى سعر الحدود للسلعة، وهو التيهي يشير إلى أثز السياسات السعرية على إنفاق المستهلكين للفني
- تطور قيمة الصادرات: بدراسة تطور قيمة الصادرات لمحصول البطاطس تبين أنها تراوحت بين حد أدنى بلغ حوالي 1. 1. مليون جنيه عام

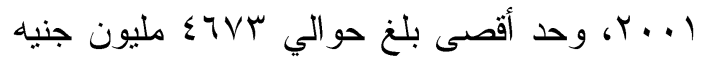

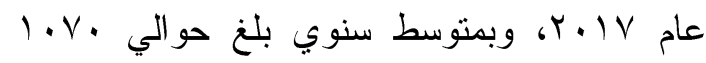

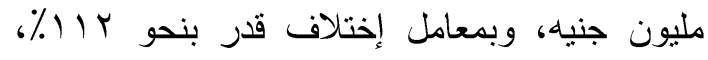
وبتقدير دالة النمو تبين أن قيمة صادرات البطاطس

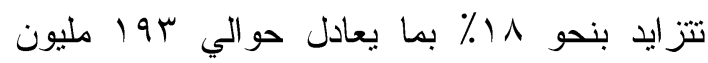

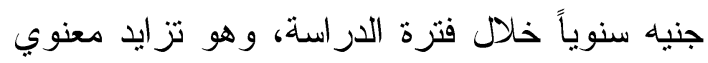
إحصائياً عند مستوى معنوية ل •,.•.

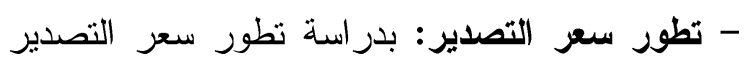

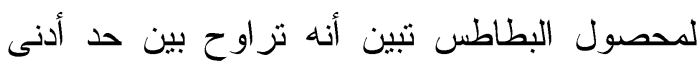

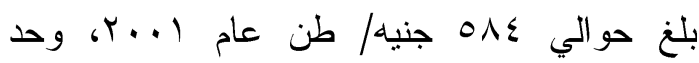

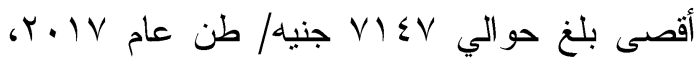
وبمتوسط سنوي بلغ حوالي سبآY جنيه/ طن،

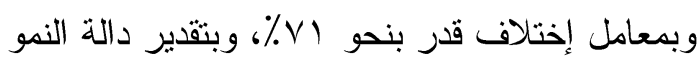

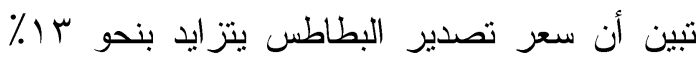

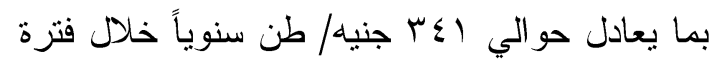

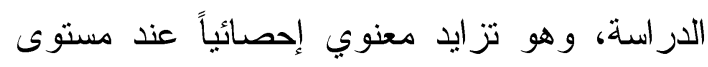
معنوية 1.,...

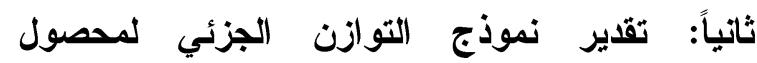

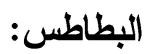
(1) مؤشرات الحماية السعرية (للمنتج أو المستهلك): يعبر معامل الحماية الأسمي للمنتج عن نسبه السعر السربه 
يرجع إلى السياسة المتبعة للدولة بفرض ضرائب

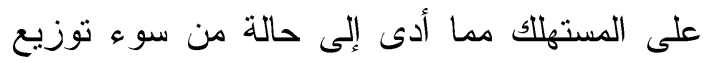
الإنفاق الاستهلاكي، ويلاحظ إرتفاع أسعار التجزئة عن أسعار الحدود.

(ج)على مستوى المجتمع: تبين من الجدول (^)، أن

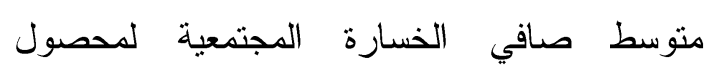

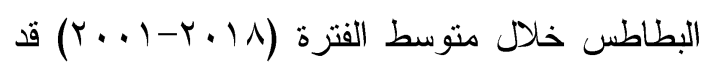

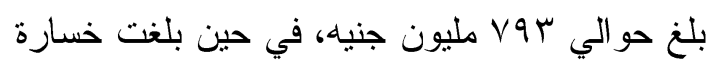

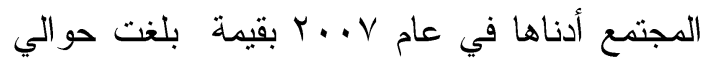

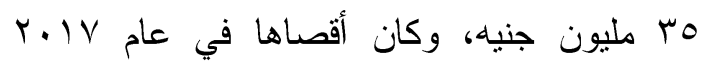

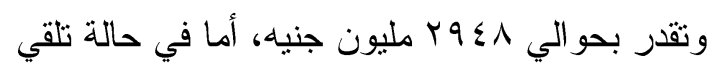
المنتجين دعماً لمحصول البطاطس بلغت أقصاها

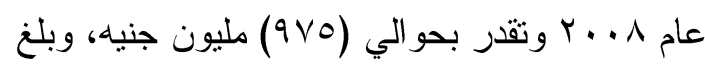

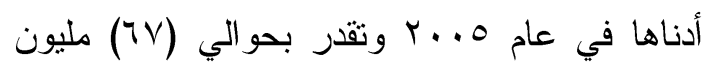
جنيه.

(ץ) مؤشر ات الرفاهية الاقتصادية:

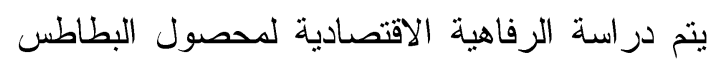

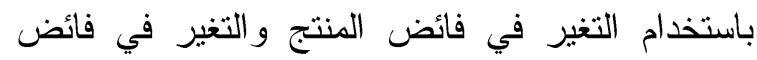
المستهلك كالآتي: (أ) التفير في فائض المنتج: يتبين من الجدول (^)، أن

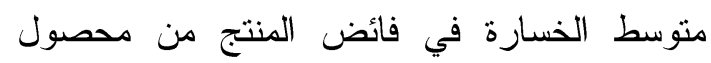

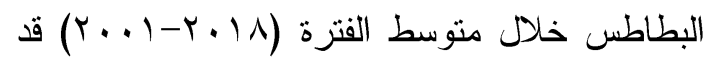
بلغت حوالي (7 ( •ـ) مليون جنيه، وتثير النتائج إلى أن الرفاهية الاقتصادية للبطاطس قد تحققت

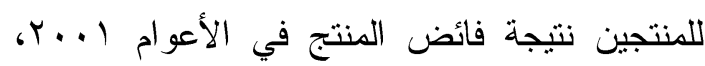
وr.... وقد برجع السبب إلى تفوق السعر المزرعي على سعر الحدود في تلاك الأعو ام فقد كان

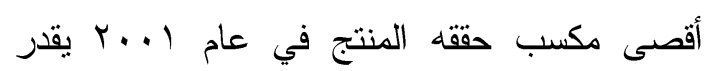

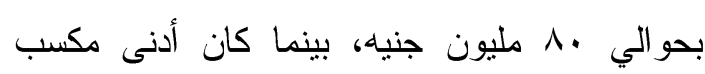

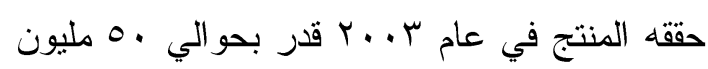

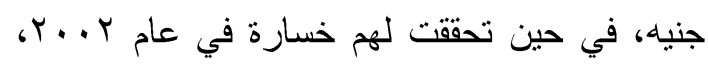

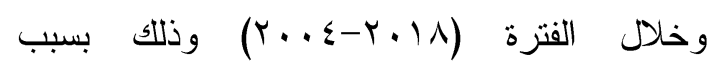
انخفاض السعر المزرعي عن سعر الحدود،
ومن ثم مستوى معيشتهم المتأثرة بأسعار التجزئة للسلع التي يستهلكونها مقارنة بأسعار الحدود، وكلما قل هذا المعامل عن الواحد الصحيح كلما كانت هذه السياسات في صالح المستهلاك و العكس صحيح. ويتضح من بيانات الجدول (^)، أن متوسط معامل

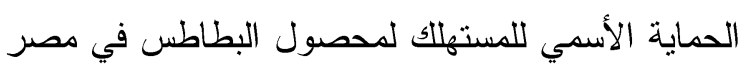

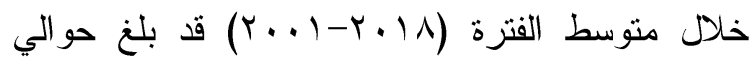
ـ7، ا، إي فرض ضرائب على المستهلك، وتكون السياسة المتبعة في غير صالح المستهلك أدى ذلك إلى فرئى إرتفاع أسعار التجزئة عن أسعار الحدود.

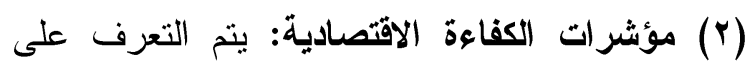

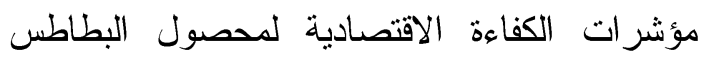
باستخدام صافي المكسب أو الخسارة الاقتصادية على مستوى المنتج ومستوى المستهلك و المستوى

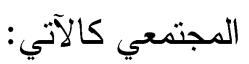
(أ) على مستوى المنتج: يتبين من الجدول (^)، أن أن

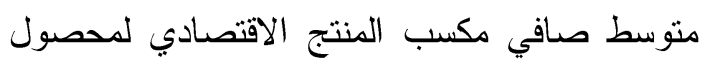
البطاطس و الذي يبين الاستخدام الاقتصادي للموارد

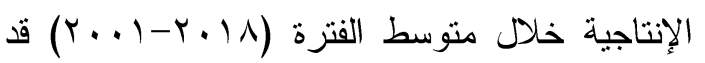
بلغ حوالي (Y7 (1) مليون جنيه، حيث بلغ أقدساها

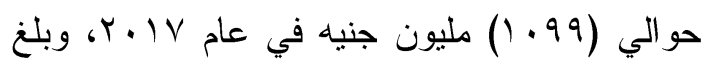

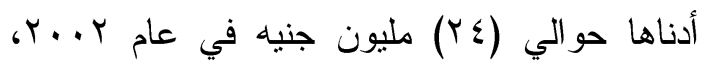
ويرجع مكسب المنتج الاقتصادي في نلك الفترة إلى لى

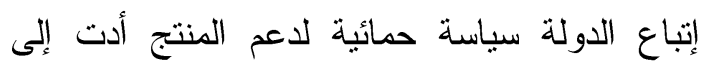
زيادة الإنتاج المحلي وبالتالي ترشيد استخدام الموارد الإنتاجية وبالتالي زيادة الكفاءة في استخدام

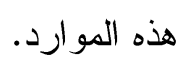

(ب)على مستوى المستهلك: تبين من الجدول (^)، أن متوسط صافي خسارة المستهلك الاقتصادية لمحصول البطاطس والذي يعكس الاستخدام الغير

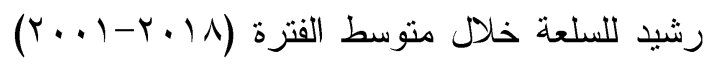
قد بلغ حوالي 900 مليون جنيه، حيث بلغ أقصاها

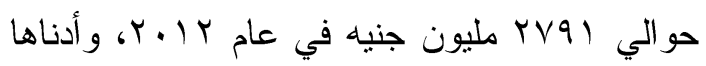

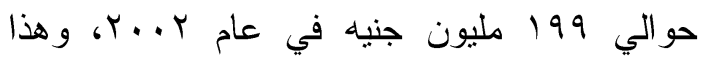


جدول V: نموذج التوازن الجزئي لمحصول البطاطس في مصر خلال القترة ( +. ب-1 I • ץ).

\begin{tabular}{|c|c|c|c|c|c|c|c|c|c|}
\hline كمية الاستهلاك عند الحدود & 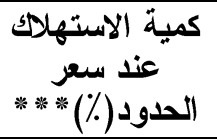 & سمية الإتتاج عند الحدود & 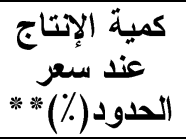 & مرونة & مرونة العرض & 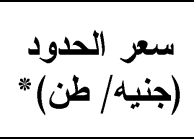 & سعر المستهليه/ طن) & سنر المنتج & السنة \\
\hline$r 00$ & T०కT4 & $r \leqslant 0-$ & $r \leq \leqslant 97-$ & $\cdot, 0 .-$ & $\cdot$, Vo & $\sum 70$ & 914 & 071 & $r \ldots l$ \\
\hline$r \leqslant 0$ & $r \leqslant \leqslant 70$ & 171 & $17 V O V$ & $.0 .-$ & $\cdot$, Vo & דיד & 994 & OVY & $r \ldots r$ \\
\hline rV & $r V T \leq r$ & $\mid r \varepsilon-$ & 1 TrA & $\cdot, 0 .-$ & $\cdot$, Vo & $09 \varepsilon$ & ITKK & $7 \leqslant 7$ & $r \ldots r$ \\
\hline r9o & Yq & VIV & VIVIr & $\cdot, 0 .-$ & $\cdot$, Vo & NTV & $1 \leqslant .0$ & . & $r \ldots \varepsilon$ \\
\hline TYY & TYYI. & 1.04 & 1.0YOV & $\cdot, 0 .-$ & $\cdot$, Vo & $9 \ldots$ & $11 Y 0$ & $7 Y \varepsilon$ & r... \\
\hline rVT & rVTr & $\varepsilon V$ & EVYY & $\cdot, 0,-$ & -, vo & 1.0 & 1709 & $\vee \wedge \varepsilon$ & $r \ldots T$ \\
\hline $\mid 11$ & $|1| \cdot 1$ & roo & To $0 \leqslant$ & $.0 .-$ & $\cdot$, Vo & ITYY & $10 \leqslant r$ & $1 \cdot \varepsilon \pi$ & $Y \ldots V$ \\
\hline $19 \mathrm{~V}-$ & $19707-$ & 1871 & IVTAYY & $.0 .-$ & $\cdot$, Vo & 11.7 & 1049 & $1.1 \mathrm{~V}$ & $r \ldots \lambda$ \\
\hline MIV- & T17119- & $\varepsilon \cdot \varepsilon r$ & $\varepsilon \cdot \varepsilon r \mu$. & $\cdot, 0 .-$ & $\cdot, \mathrm{VO}$ & rq & TrI. & 119. & $r \ldots q$ \\
\hline$r V I$ & YVITY & IVo. & $\mid V \leqslant 901$ & $\cdot, 0 .-$ & $\cdot, V_{0}$ & $r \cdots \lambda$ & TAYO & IYKT & $r \cdot 1$. \\
\hline$V \leqslant 1$ & $V \varepsilon .9 \varepsilon$ & $\{04$ & $\leqslant 07 \leqslant T$ & $\cdot, 0,-$ & $\cdot$, Vo & $10 \mathrm{VV}$ & rAo. & IrNT & $r .11$ \\
\hline VIY & $V \backslash 1 \wedge \varepsilon$ & roTr & rotrsq & $\cdot, 0 .-$ & $\cdot$, vo & TMTY & $\varepsilon \vee 7$. & $11 A r$ & $T . / Y$ \\
\hline 779 & $74 \wedge \wedge \Lambda$ & rVIV & rVIVrq & $\cdot, 0 .-$ & $\because$, Vo & r70. & 0790 & IKYT & $r .1 T$ \\
\hline $01 Y$ & $01 Y \varepsilon 1$ & §ाई & $\varepsilon \| \leqslant Y r$ & $\cdot, 0 .-$ & $\cdot$, Vo & TVIT & $\varepsilon 0 \wedge$. & $1 r r q$ & T.1 \\
\hline 511 & V) & TYYA & TYYAEA & $\cdot, 0 .-$ & $\cdot$, Vo & THYE & $\leqslant 0 \leqslant$. & $T r \varepsilon \varepsilon$ & $r .10$ \\
\hline 711 & $7 / \vee A \Lambda$ & rONV & ro1791 & $\cdot, 0 .-$ & $\cdot, V_{0}$ & YAO & ov.. & $100 Y$ & T.1T \\
\hline $1 K \Lambda-$ & ITVAq- & 71.0 & $7 \wedge . \leqslant \wedge 9$ & $\cdot, 0 .-$ & $\cdot, V_{0}$ & 079. & 019. & 191. & $r . I V$ \\
\hline rrq & $r r q \leqslant 0$ & ह1०र & $\varepsilon 10 \% \cdot \varepsilon$ & $\cdot, 0 .-$ & $\cdot, V_{0}$ & $017 \varepsilon$ & $7 \wedge 4$. & $r \leqslant \varepsilon$. & $r . / A$ \\
\hline Tr & TMY & YIY. & $r 119 \mathrm{Vr}$ & $\cdot, 0 .-$ & $\cdot$, Vo & $r \cdot \Lambda \Lambda$ & $41 \leq 9$ & $11 \leq 0$ & المتوسط \\
\hline
\end{tabular}

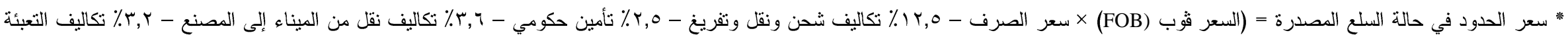
و النقل من المصنع إلى المزرعة).

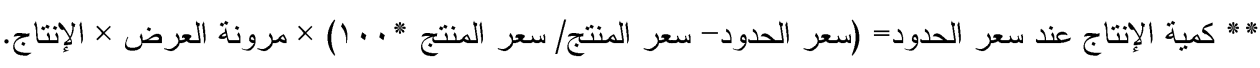

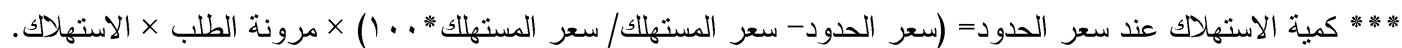

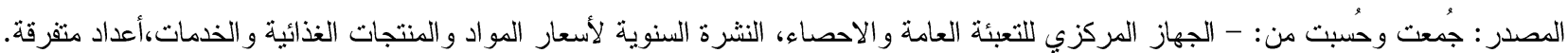

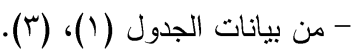




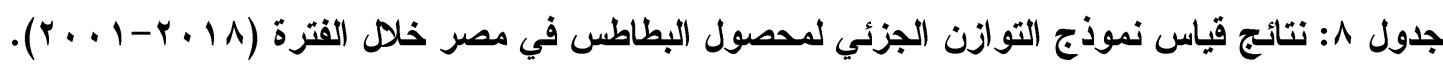

\begin{tabular}{|c|c|c|c|c|c|c|c|c|c|c|}
\hline $\begin{array}{c}\text { صافي الأثر على (لصيون جنيه) } \\
\text { NETE } \\
\text { Nلفئه }\end{array}$ & 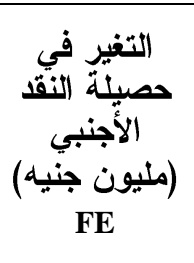 & 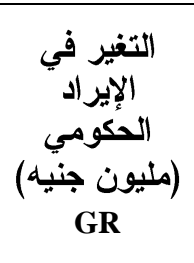 & (المئير في & أتغير في & (للاقتصادية: & اللاقتصادية & الاقتصادية & معامل الحماية & $\begin{array}{c}\text { الأسمي للمنتجائ الحماية } \\
\text { NPCP }\end{array}$ & السنة \\
\hline זוा- & $1 \ldots$ & 017 & $907-$ & A. & r4) & TOV & $1 . \pi$ & $T, I Y$ & $1, Y)$ & $r \ldots l$ \\
\hline $1 \leqslant 1-$ & $110 \mathrm{~V}$ & 7) & $710-$ & $79-$ & $1 \leqslant 1$ & 199 & $O \wedge-$ & 1,07 & $\cdot, 9$. & $r \ldots r$ \\
\hline \&1१- & IYNY & $\Lambda \wedge \wedge$ & 1 Tos- & 0. & $\left.\sum 1\right\rceil$ & r. & ov & $Y, Y \xi$ & $1, .9$ & $r \ldots r$ \\
\hline $111-$ & $10 \mathrm{NV}$ & $1 \leqslant \mu 1$ & $117 \%-$ & Кᄉ广- & 111 & Tro & YIY- & $1,7 Y$ & $\cdot, \mathrm{VT}$ & r... \\
\hline TV & 19.0 & IrVo & $V Y \varepsilon-$ & ONK- & $7 V-$ & rYo & rqY- & $1, Y 0$ & $\cdot, 79$ & r... \\
\hline \& ४ч- & IAYO & $I Y \wedge \Lambda$ & $1791-$ & ro- & $\sum \mu_{4}$ & $\leqslant 7$. & $r \varepsilon-$ & $r, .4$ & $\cdot, 9 \mathrm{~V}$ & $r \ldots T$ \\
\hline ro- & $r q \leqslant r$ & $1.0 Y$ & $1.9-$ & rVA- & ro & ro. & Y10- & $1, Y 4$ & $\cdot, 10$ & $r \ldots V$ \\
\hline $9 \vee 0$ & TrOI & 1901 & $94 \xi$ & $1918-$ & $9 \vee 0-$ & rrq- & $7 \leqslant Y-$ & $\cdot, 10$ & $\cdot, 7$. & $r \ldots \lambda$ \\
\hline$\{97$ & $11 Y \wedge-$ & \&90 & TYQT & TVO - & ₹97- & NKr- & TrV & $\cdot, \sqrt{ } \cdot 9$ & $\cdot, \varepsilon$. & $r \ldots q$ \\
\hline 111 & rVAV & $\varepsilon r V \lambda$ & r.Or- & $r \| r-$ & $111-$ & $7 r 9$ & $V \varepsilon,-$ & $1, \Gamma$ & $\cdot, 71$ & $r .1$. \\
\hline $17 \pi \varepsilon-$ & $7 / Y \varepsilon$ & $70 \leqslant V$ & VVIY- & $\leqslant 70-$ & $17 \pi \varepsilon$ & $r .11$ & rVV- & $r, \varepsilon \leqslant$ & $\cdot, \wedge \wedge$ & $r .11$ \\
\hline INr.- & TAYY & $|r+q|$ & $9 \pi 1 Y-$ & $\sum 9.9-$ & lAr. & roro & $V .0-$ & $r, \cdot r$ &., 0 & $r .1 T$ \\
\hline$r \varepsilon \cdot 1-$ & $1 \leqslant 04$ & 117998 & $1 . \varepsilon 1 .-$ & $0710-$ & $r \varepsilon=1$ & rVqI & rq.- & $r, 10$ & $\cdot, \leqslant 7$ & $r .1 T$ \\
\hline $10 . r-$ & 1ro. & $11 \leqslant 9$. & $707 Y-$ & T\&KI- & $10 . r$ & $1 \wedge T \lambda$ & ז47- & 1,79 & $\cdot, \leqslant 7$ & r.lई \\
\hline $10.9-$ & $\{.17$ & 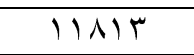 & $19.1-$ & $\varepsilon \leqslant Y)-$ & 10.9 & $r \leqslant \leqslant Y$ & qRr- & 1,90 & $\cdot, 0 \leqslant$ & $r .10$ \\
\hline $17 \leq 9-$ & $\varepsilon r 0 q$ & $\mid r r q \wedge$ & $94 \wedge V-$ & $\{$ ¿ฬו- & $17 \leqslant 9$ & YY\&K & 99 १ะ- & $r, \ldots$ & $\cdot, 0 \leqslant$ & $r .17$ \\
\hline$r q \leq \wedge-$ & $111 \mathrm{VI-}$ & 17745 & $r \cdot Y r$ & Y19.0- & $r q \leqslant \wedge$ & $797-$ & Tч\& & $\cdot, 91$ & - & $r .1 V$ \\
\hline $9 \leqslant Y-$ & $\varepsilon \backslash \vee$. & 11171 & $7991-$ & $|r \leqslant| Y-$ & $9 \leqslant Y$ & $r \cdot \xi 1$ & $1.99-$ & $1, r \mu$ & $\cdot, \leqslant V$ & $r .11$ \\
\hline VqY- & $I V \cdot \Lambda$ & $T V \leqslant 9$ & rory- & $\varepsilon .1\rceil-$ & V9Y & 900 & $17 Y-$ & $1,7 \varepsilon$ & $\cdot, 71$ & المتوسط \\
\hline
\end{tabular}

المصدر : حُسبت من: بيانات جدول (V) 
مليون جنيه في عام || (1)، وبحد أدنى بلغ حوالي

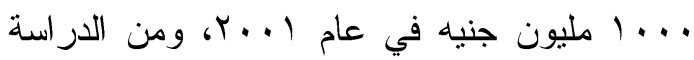

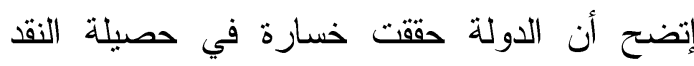
الأجنبي لمحصول البطاطس في بعض السنوات

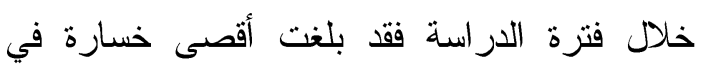
حصيلة النقا الأجنبي بحوالي (IIVI) في عام الأن Y. V

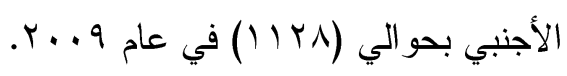

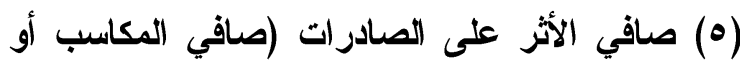

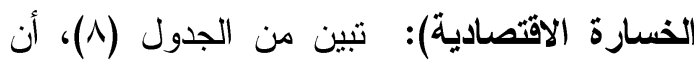

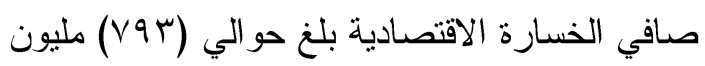

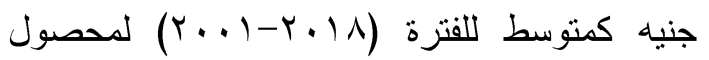

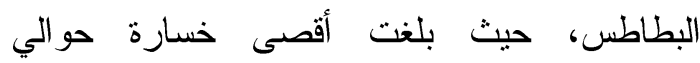

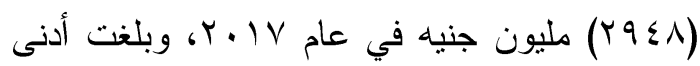

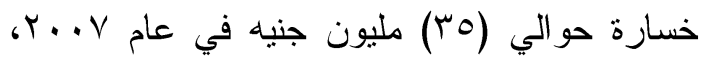

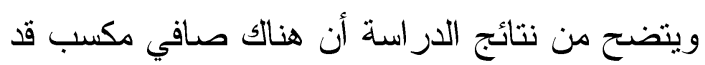

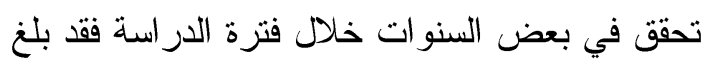
أقصى مكسب حوالي 9V0 مليون جنيه في عام

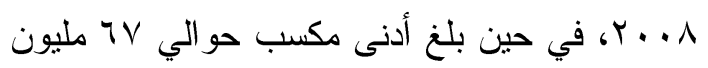

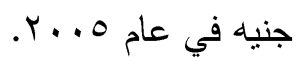

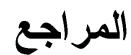

إيهاب مريد شرابين ميخائيل، دراسة اقتصادية لأهم

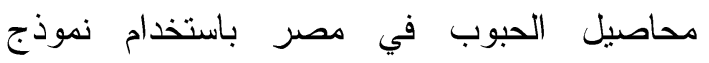

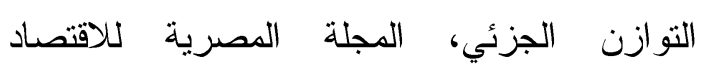

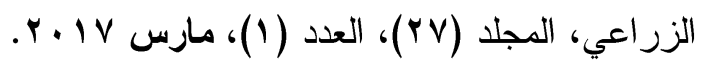
الجهاز المركزي للتعبئة العامة والاحصاء، النشرة

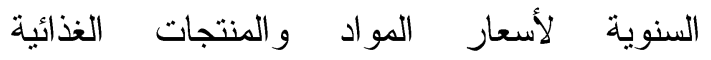
و الخدمات، أعداد متفرقة. علي عبدالجليل (وآخرون)، أثر السياسة السعرية الزراعية على إنتاج واستهلاك محصول القمح في الحولي محافظة أسيوط باستخدام نموذج التوازن الجزئي،

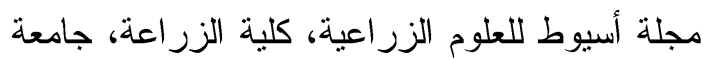

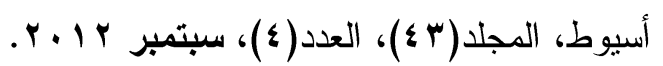

ويلاحظ أن مع إنخفاض الضرائب الضمنية يحدث إنخفاض الأعباء على المنتج و العكس صحيح، فقد كان

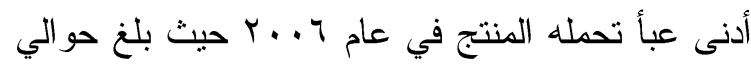

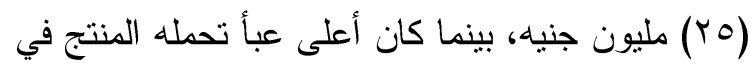

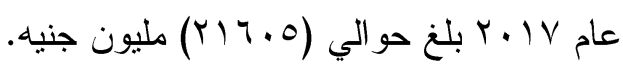
(ب) التغير في فائض المستهلك: يتبين من الجدول (^)، أن متوسط الخسارة في فائض المستهلك من فئن

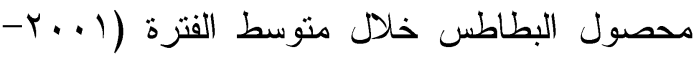

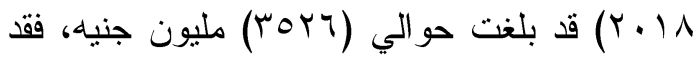

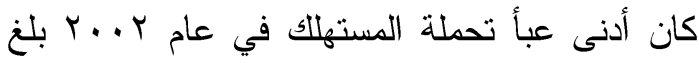
حوالي (110) مليون جنيه، بينما كان أعلى عبأ

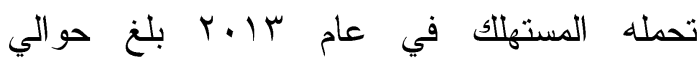

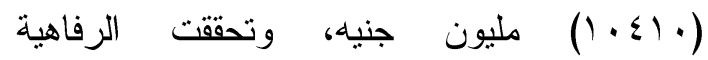
الاقتصادية للمستهكين في بعض السنوات خلال

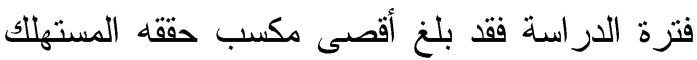

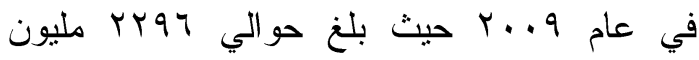

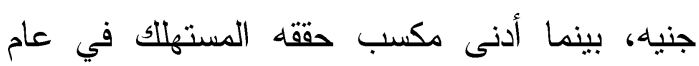

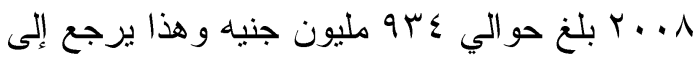
إنخفاض سعر التجزئة عن سعر الحدود خلا هذه السنو ات.

(ع) حصيلة عوائد الاولة: ويتم دراسة حصيلة عوائد الدولة لمحصول البطاطس باستخدام التغير في الإيراد الحكومي و التغير في حصبلة النقد الأجنبي كالآتي:

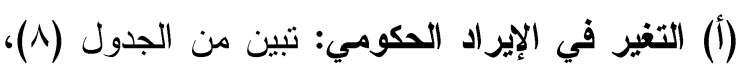
إرتفاع مكاسب الدولة في حصيلة العو ائد الحكومية الئن على الصادرات من محصول البطاطس بمتوسط بلغ

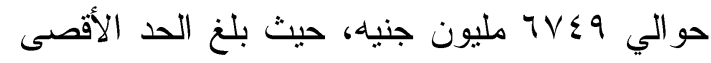

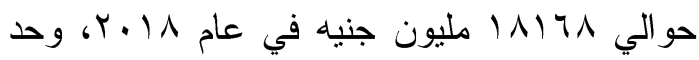

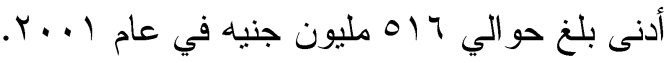

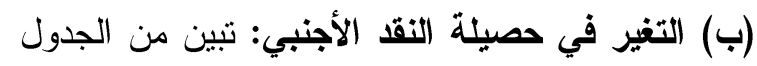

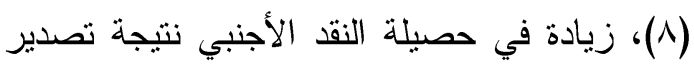
محصول البطاطس إلى الخارج بمنوسط قدر بحو الي لي لئه

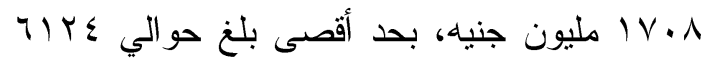




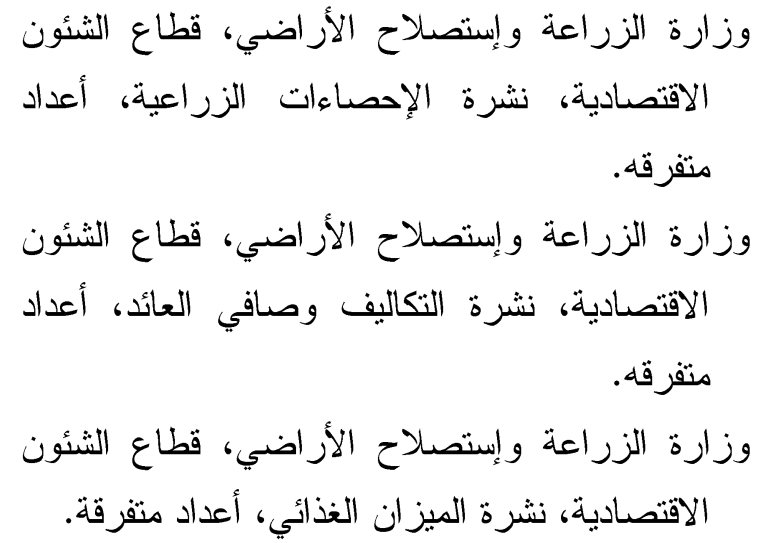

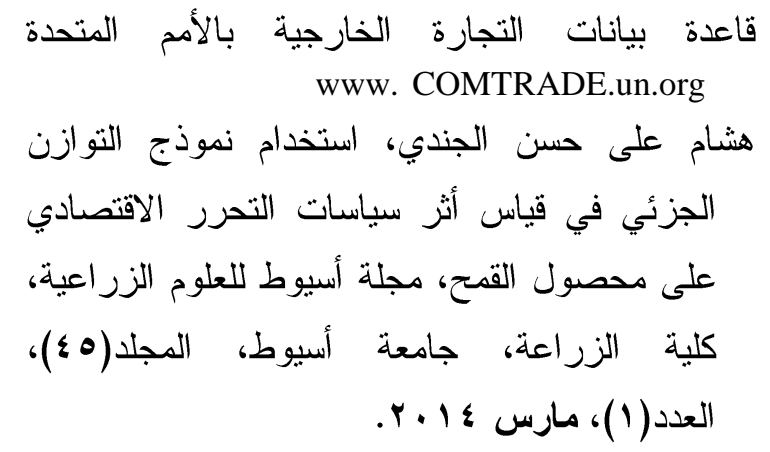

www. COMTRADE.un.org

\title{
The Impact of Pricing Policies on Potato Crop in Egypt
}

\author{
Maha Mohamed Eliwa \\ Department of Economics and Agribusiness, Faculty of Agriculture, Alexandria University
}

\begin{abstract}
The potato crop is an important food crop, as it is an important source of agricultural income and its exports contribute to the acquisition of foreign exchange, so the problem of research is limited to the impact of direct or indirect government intervention in agricultural policies pricing and productivity of potatoes, and What are the price distortions to which both potato producers and consumers in Egypt are exposed.

The research has reached some results, the most important of which are the following:

(1) The productivity indicators of both cultivated area and individual productivity, total production and consumption of the potato crop during the period (2001-2018) have taken an increasing general trend and are statistically stabilized. The economic indicators of agricultural price, revenue, costs and net yield of the potato crop have also taken an increasing general trend, the export value of the potato crop has been shown to increase by about $18 \%$, and the export price is also increasing by about $13 \%$ and the model is statistically stabilized.

(2) The study of the price policy analysis of the potato crop using the partial balance model during the average period (2001-2018) showed that the state imposes taxes on the product estimated at $32 \%$, this led to the price of the border higher than the price of the product, and also shows the imposition of taxes on the consumer, so the policy.

(3) The study indicates that there is a gain for potato crop producers during the average period of the study, which is due to the state's protectionist policy to support the product, which has led to increased domestic production and thus rationalized the use of productive resources and thus increased efficiency in the use of these resources. While it turned out that there is a net economic loss of the consumer of the potato crop, which reflects the irrational use of the commodity during the average period was about 955 million pounds, this is due to the policy adopted by the state to impose taxes on the consumer, which led to a state of poor distribution of consumer spending, and Retail prices are higher than border prices.

(4) The maximum total net loss for the potato crop in 2017 was about 2948 million pounds, while in the case of producers receiving support for the potato crop reached a maximum of about (975) million pounds in 2008. The results also indicate that the economic well-being of the potato crop has been achieved for producers as a result of the surplus of the product in 2001 and 2003, which amounted to 80 million pounds and 50 million pounds, respectively, and may be due to the superiority of the agricultural price over the border Price in those years, while the producers achieved a loss in 20022006 amounted to about (25) million pounds, while the highest burden borne by the product in 2017 was about (21605) million pounds.
\end{abstract}


(5) The average loss in consumer surplus from the potato crop during the average study period amounted to about (3526) million pounds, and based on it was the lowest burden borne by the consumer amounted to about (685) million pounds in 2002, while the highest burden borne by the consumer amounted to about (10410) million pounds in 2013, while the economic welfare of consumers about 934 in 2008 and this is due to the lower retail price than the border Price during these years mentioned.

(6) The increase in the state's gains in government revenues on exports of the potato crop was also shown during the average study period. It also shows an increase in foreign exchange earnings as a result of the export of the potato crop abroad by an average of about 1708 million pounds, but the study showed that the state achieved a loss in foreign exchange earnings for the potato crop during the study period represented in some years, namely 2009 and 2017, which amounted to (1128) million pounds and (11171) million pounds respectively.

(7) The results of the study on the impact on exports of the potato crop showed an economic loss during the average period of the study amounted to about (793) million pounds. While a net gain for potato crop exports was found in some years during the study period, the maximum gain was around EGP 975 million in 2008, while the lowest gain was around EGP 67 million in 2005.

In light of the findings, the research recommends:

(1) The need to study and modify policies on consumer support, which have led to irrational use of the potato crop and to a situation of poor distribution of consumer spending.

(2) continue to review and amend the economic reform programme, especially with regard to addressing the negative effects of the programme, namely, the reduction of taxes on agricultural producers, the increase of which leads to an increase in the net economic loss in potato crop production.

\section{الملحقات}

\begin{tabular}{|c|c|c|c|}
\hline الألتاج الكني & 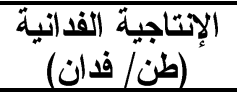 & المساحة المزفروعة فدان) & السنة \\
\hline $11 \ldots v$ & $1 \cdot, 0 \leqslant$ & $1 . \leqslant 0$ & $r \ldots 1$ \\
\hline 1170. & $1 \cdot, 7 \varepsilon$ & 1.90 & $r \ldots r$ \\
\hline 1 1 $4 \leqslant 9$ & $1 ., 0 \mathrm{~V}$ & 1197 & $r$ \\
\hline 11.19 & $11, \pi r$ & 110. & $r \ldots \varepsilon$ \\
\hline $1 \leqslant 1 V$. & $11,7 \varepsilon$ & $1 Y 1 V$ & r...o \\
\hline $1 \leq 0.0$ & $11,0 r$ & 1509 & $r . . r$ \\
\hline $1 \leqslant \vee 9$. & 11,19 & $\mid M T$ & $r \ldots V$ \\
\hline $1041 \mathrm{r}$ & 11,14 & $1 \pi \leqslant \Lambda$ & $r \ldots \Lambda$ \\
\hline $19 \leqslant \wedge r$ & 11,94 & 17rE & $r \ldots q$ \\
\hline 1801. & 11,21 & $1 \leq 97$ & $r .1$. \\
\hline $17 \vee 91$ & $11, \cdot \varepsilon$ & $10 Y Y$ & $r+11$ \\
\hline $1 V \times 17$ & 11,70 & 1041 & $r .1 r$ \\
\hline 111999 & $11, v$. & 1717 & $r .1 r$ \\
\hline $18 \cdot 19$ & 11,19 & 1047 & $r .1 \varepsilon$ \\
\hline $17 Y 77$ & 11,19 & $1 \leq 04$ & $r .10$ \\
\hline $10 V 94$ & $11, \leqslant Y$ & IrNM & $r .17$ \\
\hline $1 \leq 0.7$ & 11,10 & $1 \mathrm{H} .1$ & $r .1 \mathrm{~V}$ \\
\hline $1 \leqslant V V Y$ & $11, .9$ & سTr & $r .11$ \\
\hline $104 r 9$ & $11, Y V$ & 11007 & المتوسط \\
\hline
\end{tabular}

